\title{
De-Noising Method of Improved EEMD Algorithm Based on Cloud Simi- larity Measurement
}

\author{
Long $\operatorname{Han}^{1,2}$ and Chengwei $\mathrm{Li}^{1, *}$ \\ ${ }^{1}$ School of Electrical Engineering and Automation, Harbin Institute of Technology, Harbin, China \\ ${ }^{2}$ School of Electrical and Control Engineering, Heilongjiang University of Science and Technology, Harbin, China
}

\begin{abstract}
EEMD Algorithm is usually applied in noise reduction of rolling bearing signal because of its powerful ability in de-noising. But misjudgment in selecting sensitive IMF exists, it results in the incomplete processing of noise reduction. In order to solve this problem, this paper proposes an improved EEMD algorithm. This algorithm adopts Cloud Similarity Measurement in selecting the sensitive intrinsic mode function component which responses the fault feature. And the sensitive intrinsic mode function component is used to reconstruct signal. The simulation experiment shows that the improved EEMD algorithm has overcome the misjudgment of the original EEMD algorithm during selecting sensitive IMF, and it can do better in filtering the noise of signal. To apply the improved EEMD algorithm in de-noising of factually collected damaged AE signal, the experiment results show that it is more effective in reducing the noise interference in Acoustic Emission Signal of rolling bearing.
\end{abstract}

Keywords: AE Signal, Cloud similarity measurement, EEMD, Noise reduction.

\section{INTRODUCTION}

Rolling bearing is the most common part of the rolling mechanism, so the monitoring is really important. The technology of Acoustic emission (AE) is also important in monitoring the mode of the rolling bearing, because it can help to find the early symptom of pitting corrosion defect and the spalling of the worn surface [1-3]. Due to the terrible working environment, all the data collected from the spot has been polluted by the noises. In order to eliminate the noise of signals, some scholars bring in the technology of wavelet noise reduction, Empirical mode decomposition (EMD), Ensemble empirical mode decomposition (EEMD), and apply them in the fault diagnosis of mechanical equipment in early stage, which has achieved better results [4-6]. To use the EEMD algorithm in the de-noising of non-stationary signal is more effective than the other two algorithms, so it is usually widely applied in the process of noise reduction of rolling bearing. Although EEMD algorithm can separate and restrain the high frequency components of the noise in original signal, and the low frequency components of noise are still mixed in the IMF components, which can not be restrained well. Those reasons mentioned above lead to the situation where only part of IMFs are related to the fault information after the decomposition. Properly selecting IMFs closely related to faulty information is really significant to eliminate noises and improve the accuracy. Literatures [7-9] use sensitivity evaluation algorithm, correlation coefficient, and mutual information algorithm to select the sensitive IMFs which react on faulty characteristics from all IMFs. It

*Address correspondence to this author at the School of Electrical Engineering and Automation, Harbin Institute of Technology, Harbin, China, 150001; E-mails: 99553556@qq.com, chengweili@hit.edu.cn can eliminate the false components caused by the noise interference, and achieve certain effect in certain aspects. But misjudgment still exists, which results in the incomplete processing of noise reduction.

In view of this problem, the essay advocates the improved EEMD algorithm based on the cloud similarity measurement (CSM). The CSM of the Cloud Model is identifying the differences of the amount between clouds, it has better accuracy in similarity measurement of two time series [10]. Based on above reasons, in this paper, CSM is adopted to select the sensitive IMFs which could reflect the fault features from IMFs to reconstruct signal. And the EEMD algorithm is improved. The improved EEMD algorithm can improve the accuracy in selecting sensitive IMF and can overcome the misjudgment in the traditional algorithm. The ability of noise reduction is also improved. It achieves a good effect in de-noising the AE signal of rolling bearing.

\section{IMPROVED EEMD ALGORITHM}

\subsection{EEMD Algorithm}

EEMD algorithm is an auxiliary signal processing method dealing with noises. Using this algorithm, a Gaussian white noise is superimposed to the signals, and the mixed signals are calculated repeatedly in empirical mode decomposition. After adding the noises, the signal continuity may be achieved in different frequency regions due to the evenly distributed statistical properties of Gaussian white noise frequencies, then the IMF component model mixing degree is lowered. EEMD algorithm is shown as follows [11].

(1) The overall average number is $M$ and standard deviation of white noise is $k$.

(2) EMD experiments are executed $m$ times after adding white noise. 
I) A random Gaussian white noise $n_{m}(t)$ is added in the input signal $x(t)$, and the signal $x_{m}(t)$ is obtained,

$x_{m}(t)=x(t)+k n_{m}(t)$

II) $x_{m}(\mathrm{t})$ is decomposed by EMD, obtaining $c_{j, m} . c_{j, m}$ which stands for the $j$ th IMF obtained in the $m$ th decomposition $\left(j=1,2, \ldots, N_{m}\right) ; N_{m}$ stands for the number of IMFs in the $m$ th decomposition.

III) If $m<M$, let $m=m+1$, return 2).

IV) To take the minimum of model components in each group of IMF obtained in the $M$ times of decomposition as the final overall average number of IMF.

(3) Each IMF in $m$ times of decomposition are averaged,

$\bar{c}_{j}=\frac{\sum_{m=1}^{M} c_{j, m}}{M}\left(\mathrm{j}=1,2, \ldots, \mathrm{N}_{\mathrm{m}}, \mathrm{m}=1,2, \ldots, \mathrm{M}\right)$

(4) To output $\bar{c}_{j}$ as the $j$ th IMF obtained by EEMD decomposition. The added white noise $n_{m}(t)$ is generated randomly in each experiment. When $M$ is larger, the overall average of the added Gaussian white noise is closer to zero.

\subsection{Cloud Similarity Measurement}

Cloud similarity measurement (CSM) is made up of backward cloud generator algorithms and includes angle cosine of cloud eigenvector. The input sample point $x_{i}=\left(x_{1}\right.$, $\left.x_{2}, \ldots, x_{N}\right)$, sample point $y_{j}=\left(y_{1}, y_{2}, \ldots, y_{M}\right)$, where, $N$ and $M$ are the numbers of $x_{i}$ and $y_{j}$, respectively. The steps are as follows [12].

(1) Sample mean $\bar{X}=\frac{1}{n} \sum_{i=1}^{n} x_{i}$ is obtained based on the
mple point $x_{i}$

The first order of sample absolute center distance is $\underline{\frac{1}{n} \sum_{i=1}^{n}\left|x_{i}-\bar{X}\right|} ;$ sample variance is $S^{2}=\frac{1}{n-1} \sum_{i=1}^{n}\left(x_{i}-\bar{X}\right)^{2}$.

(2) To calculate expected value

$E_{x}=\bar{X}$

(3) To calculate entropy

$E_{n}=\sqrt{\frac{\pi}{2}} \times \frac{1}{n} \sum_{i=1}^{n}\left|x_{i}-E_{x}\right|$

(4) To calculate hyper entropy

$H_{e}=\sqrt{S^{2}-E_{n}^{2}}$

(5) To calculate cloud vector $\vec{v}_{i}=\left(E x_{i}, E n_{i}, H e_{i}\right)$ of sample point $x_{i}$ and cloud vector $\vec{v}_{j}=\left(E x_{j}, E n_{j}, H e_{j}\right)$ of sample point $y_{j}$.

(6) The similarity of any two samples $x_{i}$ and $y_{j}$ may be expressed by the included angle cosine between $\vec{v}_{i}$ and $\vec{v}_{j}$, as follows.

$$
\operatorname{sim}(i, j)=\cos \left(\vec{v}_{i}, \vec{v}_{j}\right)=\frac{\vec{v}_{i} \times \vec{v}_{j}}{\left\|\vec{v}_{i} \mid\right\| \vec{v}_{j} \|}
$$

To set similarity threshold $\delta=0.95$, retaining $\mathrm{IMF}_{j}$ when $\cos \left(\vec{v}, \vec{v}_{j}\right) \geq 0.95$, others are removed.

\subsection{Improved EEMD Algorithm}

The improved EEMD algorithm can be summarized in the following steps.

(1) To calculate vectors $\vec{v}=(E x, E n, H e)$ of signal $v$ by backward cloud generator algorithm.

(2) To decompose the signal $v$ by EEMD, obtaining $\mathrm{IMF}_{j}$ $(j=1,2, \ldots, n)$.

(3) To calculate vectors $\vec{v}_{j}=\left(E x_{j}, E n_{j}, H e_{j}\right)$ of signal $\mathrm{IMF}_{j}$ by backward cloud generator algorithm.

(4) To calculate $\cos \left(\vec{v}, \vec{v}_{j}\right)=\frac{\vec{v} \times \vec{v}_{j}}{\|\vec{v}\|\left|\vec{v}_{j}\right|}$, making sure of cloud vector of signal $v$ and the similarity degree of cloud vector of $\mathrm{IMF}_{j}$.

(5) To set the similarity threshold $\delta=0.95$, then to select the $\mathrm{IMF}_{j}$ of $\cos \left(\vec{v}, \vec{v}_{j}\right) \geq 0.95$ as sensitive IMF to reconstruct the signal.

\section{THE COMPARISON OF ALGORITHM}

D. Mitrakovic and other people use damped exponential signals of three different frequencies and different attenuation coefficients to simulate AE signal. The signal model is given as follows[13].

$\left.f(x)=\sum_{i=1}^{3} A_{i} e^{\left[-a_{i}\left(t-t_{i}\right)^{2}\right.}\right] \sin \left[2 \pi f_{i}\left(t-t_{i}\right)\right]$

In this equation, $A_{i}, a_{i}, t_{i}, f_{i}$ are the $i$ th harmonic signal amplitude, attenuation coefficient, peak instant and the characteristic frequency, respectively. The parameters of typical AE signal are valued as follows, $A_{i}=2(i=1,2,3), a_{1}=6.24 \times 10^{8}$, $a_{2}=1.56 \times 10^{8}, a_{3}=2.79 \times 10^{8}, t_{1}=0.4 \mathrm{~ms}, t_{2}=0.6 \mathrm{~ms}, t_{3}=0.8 \mathrm{~ms}$, $f_{1}=70 \mathrm{KHz}, f_{2}=60 \mathrm{KHz}, f_{3}=80 \mathrm{KHz}$. In fact, most of the noise signals are white noises. So according to the formula of sampling frequency $f=500 \mathrm{kHz}$, the white Gaussian noise whose standard deviation is 0.246 is added into the stimulated AE signal, and the waveform is shown in Fig. (1). The EEMD algorithm and the improved EEMD algorithm is proposed to test the noise reduction of simulated AE signal.

In EEMD decomposition, the IMF1 IMF4 were obtained, as shown in Fig. (2). The added noise amplitude is 0.01 times of the standard deviation of signal, and the overall average time is 200 .

As can be seen in Fig. (2), the IMF1 and IMF2 decomposed by EEMD are actual original signal component in which the noise interference is eliminated. IMF3 and IMF4 are meaningless false components, which will be excluded in subsequent analysis. The comparison between the mutual information and cloud similarity of each IMF and original signal is shown in Table 1. According to the literature [14], the calculated mutual information threshold is 0.0357 . As shown in Table 1, IMF1, IMF2 and IMF3 are considered to be actual components for their thresholds bigger than 0.0357 . Only the IMF4 component is removed as false components. 
Therefore, it is easy to cause misjudgment when the mutual information eliminates false component. From the threshold $\delta=0.95$ of CSM, the threshold value of IMF1 and IMF2 in CSM is bigger than $\delta$, so it is maintained as the actual components, and the false components IMF3 and IMF4 are removed. It is coincided with the situation in Fig. (2). Therefore, it is effective to select sensitive IMF by the CSM, which has better accuracy than the mutual information method, overcoming the misjudgment.

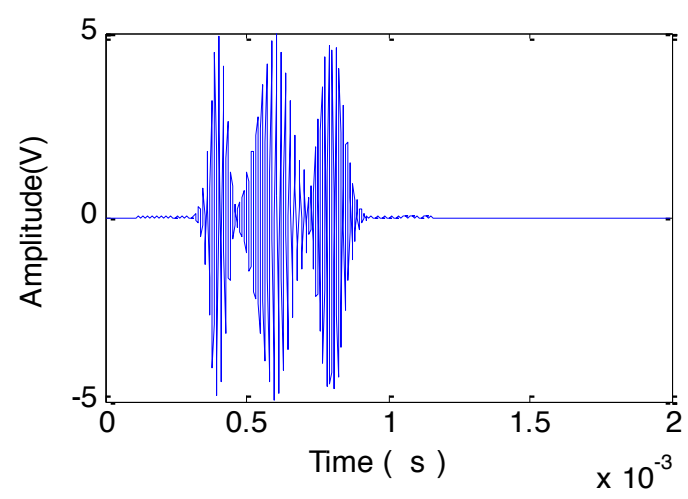

(a) Original AE signal

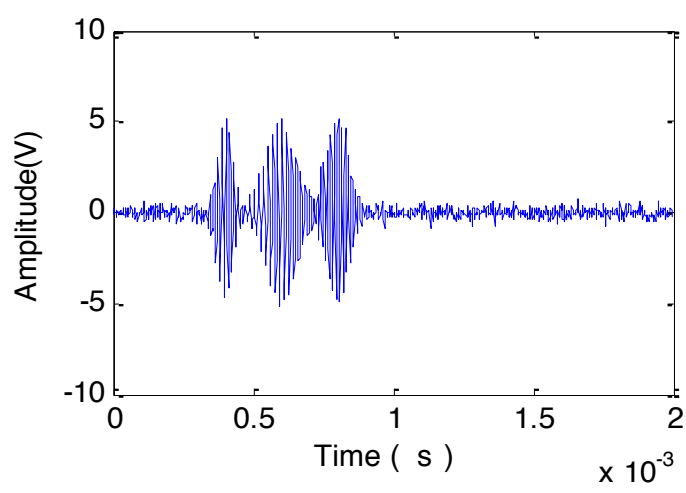

(b) Adding noise AE signal

Fig. (1). Simulated AE signal waveform.
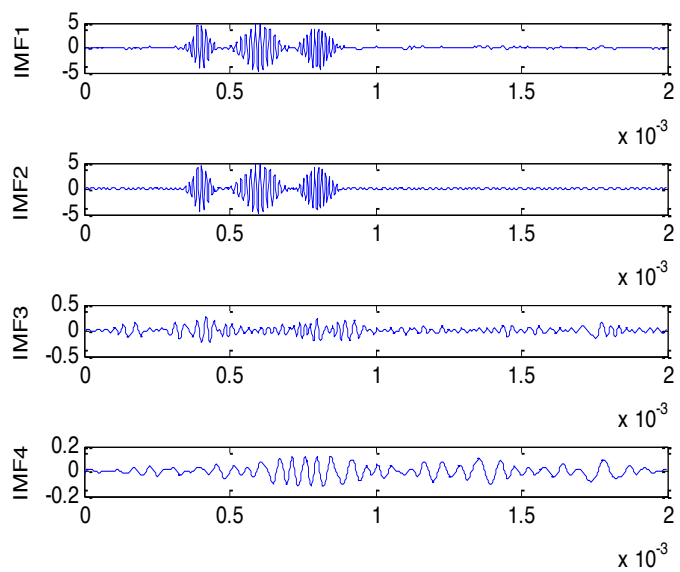

Fig. (2). IMF1 IMF4 Obtained in EEMD Decomposition.
Table 1. Comparison between the mutual information and cloud similarity of each IMF and original signal.

\begin{tabular}{|c|c|c|c|c|}
\hline & IMF1 & IMF2 & IMF3 & IMF4 \\
\hline \hline Mutual Information & 0.1175 & 0.0998 & 0.0363 & 0.028 \\
\hline Cloud Similarity & 0.9653 & 0.9551 & 0 & 0 \\
\hline
\end{tabular}

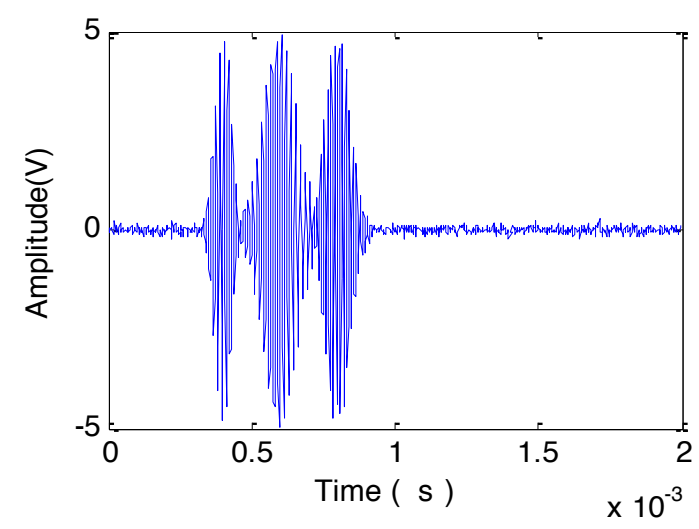

(a) Reconstructed signal by EEMD

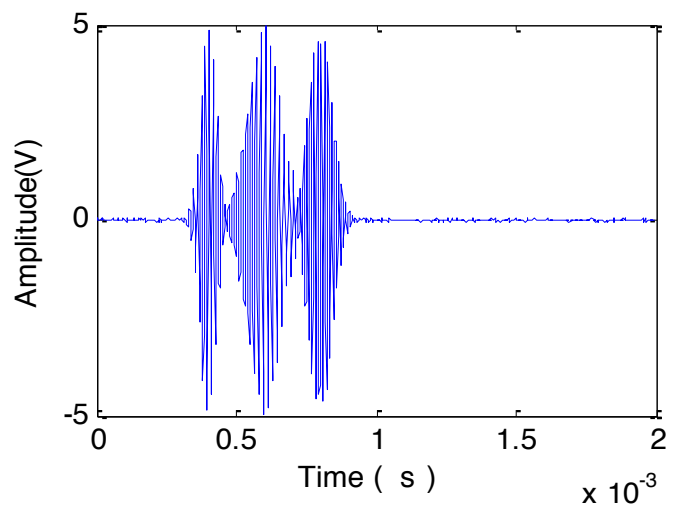

(b) Reconstructed signal by Improved EEMD

Fig. (3). Reconstructed AE signal waveform.

As shown in Fig. (3), the reconstructed signal calculated by improved EEMD algorithm is more smooth than that of the traditional EEMD algorithm. It is more closer to original signal, and the effect of de-noising is better. In order to further compare the effect in de-noising between improved EEMD algorithm and traditional EEMD algorithm, white Gaussian noise is added into the original signal respectively with different standard deviations of 0.497、0.748 and 0.996, to get the evaluation of SNR and the MSE of the two methods in de-noising, as shown in Table 2.

To see from Table 2, the SNR of the improved EEMD algorithm is higher when it is added with white Gaussian noise with different standard deviation. The MSE is smaller, so the effect of noise reduction calculated by improved EEMD algorithm is better than the traditional EEMD algorithm. 
Table 2. Evaluation of De-noising results with two ways.

\begin{tabular}{|c|}
\hline $\begin{array}{c}\text { EEMD Improved EEMD } \\
\text { SNR of Adding }\end{array}$ \\
Noise Signal SNR MSE SNR MSE \\
\hline \hline 13.6123 .320 .006441 .580 .0032 \\
7.4223 .250 .006841 .560 .0034 \\
3.8323 .230 .006841 .550 .0034 \\
1.5423 .230 .006841 .550 .0034 \\
\hline
\end{tabular}

\section{PROJECT APPLICATION}

The simulated bearing's inner ring damage experiment was finished on the rolling bearing's experiment rig. The K001 rolling bearing is used, then we can get the damaged sample of bearing by using electron discharge machining (EDM). The facility is the four-channel signal acquisition system PCI-2-PAC which is produced by an American Physical acoustics company, the type of transducer is R15, 2/4/6 pre-amplifiers were selected, and the selected gain is $40 \mathrm{~dB}$. During the experiment, the motor speed is $14000 \mathrm{r} / \mathrm{min}$, and the signal sampling rate is 500KSPS.

Fig. (4) (a) and (b) show the time domain waveform and the spectrogram, which are measured by the transducer during the rolling bearing's inner ring damage. Fig. (4)(c) (f) show the time domain waveform and spectrogram, which denoise AE signal calculated by improved EEMD and traditional EEMD algorithm.

To see from Fig. (4), the prominent frequency of AE signal before de-noising is widely distributed in the Spectrum map, the frequency band which contains noise is wide. The Spectrum of signal which is de-noised with the improved EEMD algorithm is more concentrated than that of the traditional EEMD algorithm. A certain kind of noise signals in some frequency band are restrained.

In order to further compare the effect of the two ways in de-noising of actual testing signal, SNR, MSE and smoothness are applied in evaluating. The comparison of evaluating index is shown in Table 3.

Table 3. Comparison of Evaluating Index.

\begin{tabular}{|c|c|c|c|}
\hline Method & SNR & MSE & Smoothness \\
\hline \hline EEMD & 12.56 & 0.00196 & 0.0178 \\
\hline Improved EEMD & 27.82 & 0.00083 & 0.0086 \\
\hline
\end{tabular}

Seen from Table $\mathbf{3}$, the effect of de-noising calculated by improved EEMD algorithm is obviously better than that of traditional EEMD algorithm. The SNR is the biggest; the MSE is the smallest and the smoothness is the best. The above experimental results show the effectiveness of improved EEMD algorithm in noise reduction.

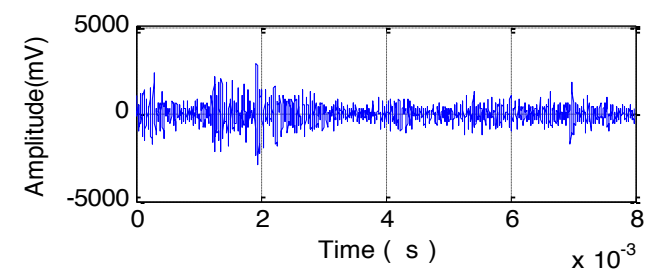

(a) Time-domain waveform of original damaged signal.

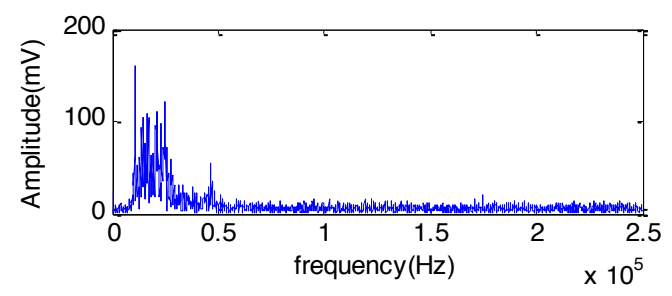

(b) Spectrogram of original damaged signal.

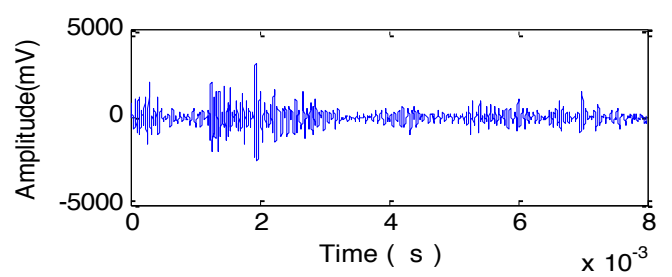

(c) Time domain waveform of signal which is filtered by EEMD algorithm

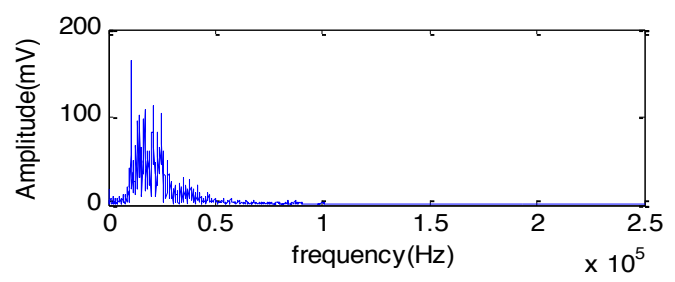

(d) Spectrogram of signal which is filtered by EEMD algorithm

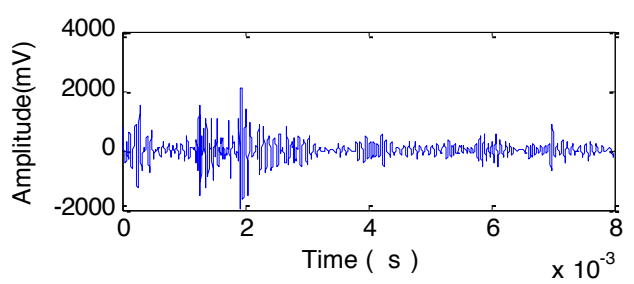

(e) Time domain waveform of signal which is filtered by improved EEMD algorithm

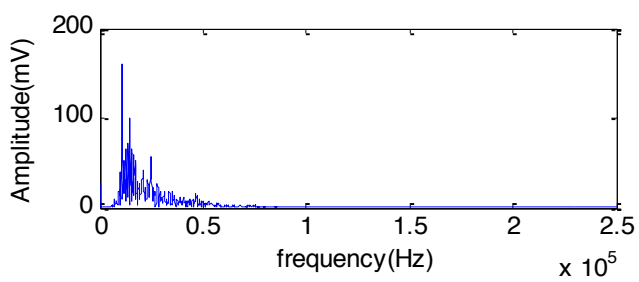

(f) Spectrogram of signal which is filtered by improved EEMD algorithm

Fig. (4). Time-domain waveform and the spectrum of AE signal before and after filtering. 


\section{CONCLUSION}

This paper advocates that the EEMD algorithm is improved by using Cloud Similarity Measurement in selecting sensitive IMF components to reconstruct signal. The improved EEMD algorithm has overcome the misjudgment made by the original EEMD algorithm during its selecting sensitive IMF, and it has improved the accuracy of reconstructing signal. In simulation experiment, the improved EEMD algorithm is better than original EEMD algorithm by comparing the SNR with MSE. Two methods of noise reduction analyze the actual collected damaged AE signal, which proves that the improved EEMD algorithm is stronger in noise reduction, and it can improve the identification ability of the AE signal in rolling bearing.

\section{CONFLICT OF INTEREST}

The authors confirm that this article content has no conflict of interest.

\section{ACKNOWLEDGEMENTS}

The research was supported partially by National Natural Science Foundation of China (51107015), The Science and technology research projects of the Education Department of Hei Long Jiang Province (12543057)

\section{REFERENCES}

D. Mba, and B.K.N. Rao, "Development of acoustic emission technology for condition monitoring and diagnosis of rotating machines: bearings, pumps, gearboxes, engines, and rotating structures," The Shock and Vibration Digest, vol. 38, pp. 3-16, 2006.
[2] N. Tandon, G.S. Yadava, and K.M. Ramakrishna, "A comparison of some condition monitoring techniques for the detection of defect in induction motor ball bearings," Mechanical systems and signal processing, vol. 21, pp. 244-256, 2007.

[3] A.M. AlGhamdi, and D. Mba, "A Comparative experimental study on the use of acoustic emission and vibration analysis for bearing defect identification and estimation of defect size," Mechanical Systems and Signal Processing, vol. 20, pp. 1537-1571, 2012.

[4] C.H. Jiang, L.S. Wang, and W. You, "Research on acoustic emission signal de-noising based on translation invariant wavelet," Chinese Journal of Scientific Instrument, vol. 27, pp. 2004-2010, 2006.

[5] H. Li, H. Zheng, and L. W. Tang, "Application acoustic emission and empirical mode decomposition to faults diagnosis of bearing," Proceedings of the CSEE, vol. 26, pp. 24-128, 2006.

[6] Z. H. Wu, and N. E. Huang, "Ensemble empirical mode decomposition: a noise assisted data analysis method," Advances in Adaptive Data Analysis, vol. 1, pp. 1-41, 2009.

[7] Y. G. Lei, "Machinery fault diagnosis based on improved hilberthuang transform," Journal of Mechanical Engineering, vol. 47, pp. 71-77, 2011

[8] Z. Zhou, Y. S. Zhu, and Y. Y Zhang, "Adaptive fault diagnosis of rolling bearings based on EEMD and demodulated resonance," Journal of Vibration and Shock, vol. 32, pp. 76-80, 2012.

[9] Y. Shen, L. Wang, and Z. Zhao, "Fault diagnosis for rolling bearing of wind turbine based on improved HHT," Measurement and Control Technology, vol. 32, pp. 40-44, 2013.

[10] D. Li, and D. Yi. "Artificial intelligence with uncertainty," Bei jing: National Defense Industry Press, pp. 143-185, 2005.

[11] Z. Jian,Y. R. Qiang, and R. X. Gao, "Performance enhancement of ensemble empirical mode decomposition," Mechanical Systems and Signal Processing, vol. 2, pp. 1018-1026, 2010.

[12] D. Xu, and $\mathrm{X}$. Li, "Recommendation algorithm of item ratings prediction based on cloud, "Computer Engineering, vol. 36: 48-50, 2010.

[13] D. Mitrakovic, I. Grabec, and S. Sedmak, "Simulation of AE signals and signal analysis systems ," Ultrasonic, vol. 9, pp. 227-232, 1985.

[14] A.P. Albert, and A.O. Nii, "A criterion for selecting relevant intrinsic mode functions in empirical mode decomposition," Advances in Adaptive Data Analysis, vol. 2, pp. 1-24, 2010.

Received: May 26, 2015

Revised: July 14, 2015

Accepted: August 10, 2015

(C) Han and Li; Licensee Bentham Open.

This is an open access article licensed under the terms of the (https://creativecommons.org/licenses/by/4.0/legalcode), which permits unrestricted, noncommercial use, distribution and reproduction in any medium, provided the work is properly cited. 